\title{
Effect of penicillin-based antibiotics, amoxicillin, ampicillin, and piperacillin, on drug-metabolizing activities of human hepatic cytochromes P450
}

\author{
Toshiro Niwa, Mari Morimoto, Takako Hirai, Tomomi Hata, Misato Hayashi \\ and Yurie Imagawa
}

School of Pharmacy, Shujitsu University, 1-6-1 Nishigawara, Naka-ku, Okayama 703-8516, Japan

(Received October 17, 2015; Accepted December 5, 2015)

\begin{abstract}
The effects of three kinds of penicillin-based antibiotics, amoxicillin, ampicillin, and piperacillin, on drug-metabolizing activity of human hepatic cytochrome P450 (P450 or CYP) were investigated. Metabolic activities of P450s expressed in recombinant Escherichia coli at substrate concentrations around the Michaelis constant were compared in the presence or absence of the antibiotics. Amoxicillin, ampicillin, and piperacillin at 0.5 or $1 \mathrm{mM}$ concentrations neither inhibited nor stimulated CYP2C9mediated tolbutamide methylhydroxylation, CYP2D6-mediated dopamine formation from $p$-tyramine, or CYP3A4- or CYP3A5-mediated testosterone 6 $\beta$-hydroxylation. However, amoxicillin and piperacillin inhibited CYP2C8-mediated aminopyrine $N$-demethylation at $50 \%$ inhibitory concentration of 0.83 and $1.14 \mathrm{mM}$, respectively. These results suggest that piperacillin might inhibit CYP2C8 clinically, although the interactions between these three penicillin-based antibiotics and other drugs that are metabolized by P450s investigated would not be clinically significant.
\end{abstract}

Key words: Penicillin-based antibiotics, Amoxicillin, Ampicillin, Piperacillin, Human hepatic cytochrome P450, Drug-drug interaction

\section{INTRODUCTION}

Cytochrome P450s (P450 or CYP) are a superfamily of enzymes that catalyze the oxidation of a wide variety of endogenous and exogenous chemicals, including drugs, carcinogens, and steroids (Rendic, 2002; Williams et al., 2004; Niwa et al., 2015). CYP2C9, CYP2D6, and CYP3A4/5 account for 10\%-20\%, 2\%-9\%, and $30 \%-40 \%$, respectively, of total $\mathrm{P} 450 \mathrm{~s}$ in human hepatic microsomes (Shimada et al., 1994; Imaoka et al., 1996) and are responsible for $20 \%, 20 \%-30 \%$, and $50 \%$, respectively, of the oxidation of all pharmaceuticals used by humans (Williams et al., 2004; Funae et al., 2003). Thus, CYP3A4 is generally recognized as the predominant form expressed in the human liver and intestine, whereas CYP3A5 is expressed polymorphically (Niwa et al., 2008). Multiple drug therapy is a common therapeutic practice, particularly in patients with several diseases or conditions, and many drug-drug interactions involving metabolic inhibition have been reported. Because antibiotics are co-administered in most cases, the possibility of interactions between them and other drugs exists (von Rosensteil and Adam, 1995; Fuhr et al., 1992). It is well known that macrolide antibacterials, especially troleandomycin and erythromycin, are inhibitors of CYP3A4mediated metabolism (Rendic, 2002; von Rosensteil and Adam, 1995). Quinolone antibacterial agents, including enoxacin and ciprofloxacin, inhibit CYP1A2-mediated metabolism of drugs (Rendic, 2002; Fuhr et al., 1992). In contrast, cefixime and cefdinir, oral cephalosporins, neither inhibited nor stimulated drug-metabolizing activities of P450s (Niwa et al., 2004).

The penicillin-based antibiotics, amoxicillin, ampicillin, and piperacillin, have broad and potent activity against various pathogens, including gram-positive organisms such as Staphylococcus and Streptococcus species (Wright, 1999; Zosyn Package Insert, 2015; Sawacillin Package Insert, 2015; Unasyn Package Insert, 2015). Piperacillin is partially metabolized by CYP2C8, followed by CYP2A6 and CYP2C9, to form the deethylated metabolite (Zosyn Package Insert, 2015). However, there have been a few reports describing not only the con-

Correspondence: Toshiro Niwa (E-mail: tniwa@shujitsu.ac.jp) 
tribution of P450s to the metabolism of penicillin-based antibiotics (except for piperacillin) but also the effect of these antibiotics on the drug metabolizing activity of human hepatic P450. In the present study, we investigated the effects of amoxicillin, ampicillin, and piperacillin on specific activities of recombinant CYP2C8, CYP2C9, CYP2D6, CYP3A4, and CYP3A5.

\section{MATERIALS AND METHODS}

\section{Materials}

P450s expressed in recombinant Escherichia coli (Bactosomes) were obtained from Cypex Ltd. (Dundee, UK). Amoxicillin, 6 $\beta$-hydroxytestosterone, and tolbutamide were purchased from Sigma-Aldrich (St Louis, MO, USA). Piperacillin sodium, $p$-tyramine hydrochloride, and 3-hydroxytyramine (dopamine) hydrochloride were obtained from Tokyo Chemical Industry (Tokyo, Japan). Ampicillin sodium and testosterone were purchased from Nakarai Tesque (Kyoto, Japan). All other reagents and organic solvents used were of the highest purity commercially available.

\section{Determination of human drug-metabolizing P450 activities}

Aminopyrine $N$-demethylation (CYP2C8), tolbutamide methylhydroxylation (CYP2C9), dopamine formation (CYP2D6), and testosterone 6 $\beta$-hydroxylation (CYP3A4 and CYP3A5) were determined in the presence or absence of amoxicillin, ampicillin, and piperacillin, as described previously (Niwa et al., 1999, 2004, 2005 and 2007). The incubation mixture consisted of human P450, $1 \mathrm{mM}$ NADPH, and $100 \mathrm{mM}$ potassium phosphate buffer ( $\mathrm{pH}$ 7.4 ) in a final volume of $0.5 \mathrm{~mL}$. The $\mathrm{P} 450$ concentrations in the mixture were 10 (for aminopyrine $N$-demethylation, dopamine formation, and testosterone $6 \beta$-hydroxylation) and $40 \mathrm{pmol} / \mathrm{mL}$ (for tolbutamide methylhydroxylation). Incubation times were 5 (for aminopyrine $N$-demethyla- tion and testosterone $6 \beta$-hydroxylation), 10 (for dopamine formation), or $60 \mathrm{~min}$ (for tolbutamide methylhydroxylation). Concentrations of aminopyrine, tolbutamide, $p$-tyramine, and testosterone were 5,000, 400, 60, and $60 \mu \mathrm{M}$, respectively; which are around the expected $\mathrm{K}_{\mathrm{m}}$ (Niwa et al., 1999, 2004, 2005 and 2007). All data were analyzed using the average of triplicate determinations. In the preliminary experiments, the linearity of reaction with P450 concentration and incubation time was confirmed for each P450. Fifty percent inhibitory concentration $\left(\mathrm{IC}_{50}\right)$ was calculated using nonlinear regression by means of JMP 5 software (SAS Institute Inc., Cary, NC, USA). Statistical significance was determined by Student's $t$-test, and the significance level was set at $p<0.05$.

\section{RESULTS}

The inhibitory effects of amoxicillin, ampicillin, and piperacillin on drug-metabolizing activities of recombinant P450s are summarized in Table 1. The metabolic activities of recombinant $\mathrm{P} 450 \mathrm{~s}$ in the absence of penicillin-based antibiotics were similar to those reported previously (Niwa et al., 1999, 2004, 2005 and 2007). CYP2C9-mediated tolbutamide methylhydroxylation, CYP2D6-mediated dopamine formation, and CYP3A4 and CYP3A5-mediated testosterone 6 $\beta$-hydroxylation in the presence of the penicillin-based antibiotics at a concentrations of $0.1-1 \mathrm{mM}$ were $87 \%-111 \%$ of control, indicating that these penicillin-based antibiotics had neither inhibitory nor stimulatory effects against these P450s. Because it was demonstrated that piperacillin is metabolized by CYP2C8 (Zosyn Package Insert, 2015), the effect of penicillin-based antibiotics on CYP2C8-mediated aminopyrine $\mathrm{N}$-demethylation were investigated using various concentration of the antibiotics (Fig. 1). At $1 \mathrm{mM}$ concentration, amoxicillin, ampicillin, and piperacillin inhibited CYP2C8 activity by $55.8 \%, 17.0 \%$, and $46.0 \%$, respectively. The estimated $\mathrm{IC}_{50}$ values for amoxicillin

Table 1. Effect of amoxicillin, ampicillin, and piperacillin on drug-metabolizing activities of recombinant P450s.

\begin{tabular}{|c|c|c|c|c|c|c|c|c|}
\hline \multirow{3}{*}{ P450 } & \multirow{3}{*}{ Reaction } & \multirow{3}{*}{$\begin{array}{c}\text { Control activity } \\
(\mathrm{nmol} / \mathrm{min} / \mathrm{nmol} \mathrm{P} 450)\end{array}$} & \multicolumn{6}{|c|}{$\%$ of control activity } \\
\hline & & & \multicolumn{2}{|c|}{ Amoxicillin } & \multicolumn{2}{|c|}{ Ampicillin } & \multicolumn{2}{|c|}{ Piperacillin } \\
\hline & & & $0.1 \mathrm{mM}$ & $0.5 \mathrm{mM}$ & $0.1 \mathrm{mM}$ & $1 \mathrm{mM}$ & $0.1 \mathrm{mM}$ & $1 \mathrm{mM}$ \\
\hline CYP2C9 & Tolbutamide methylhydroxylation & $1.01 \pm 0.18$ & $95 \pm 4$ & $99 \pm 8$ & $101 \pm 0$ & $110 \pm 0$ & $106 \pm 24$ & $100 \pm 28$ \\
\hline CYP2D6 & Dopamine formation & $3.82 \pm 0.23$ & $107 \pm 8$ & $105 \pm 6$ & $100 \pm 0$ & $111 \pm 10$ & $100 \pm 8$ & $92 \pm 16$ \\
\hline CYP3A4 & Testosterone $6 \beta$-hydroxylation & $39.7 \pm 4.7$ & $98 \pm 10$ & $106 \pm 15$ & $87 \pm 7$ & $94 \pm 21$ & $101 \pm 0$ & $103 \pm 12$ \\
\hline CYP3A5 & Testosterone $6 \beta$-hydroxylation & $49.9 \pm 6.2$ & $97 \pm 8$ & $88 \pm 15$ & $88 \pm 5$ & $87 \pm 10$ & $88 \pm 1$ & $94 \pm 15$ \\
\hline
\end{tabular}

Values are means \pm S.D. of triplicate experiments. 
Effect of penicillin-based antibiotics on human hepatic P450s

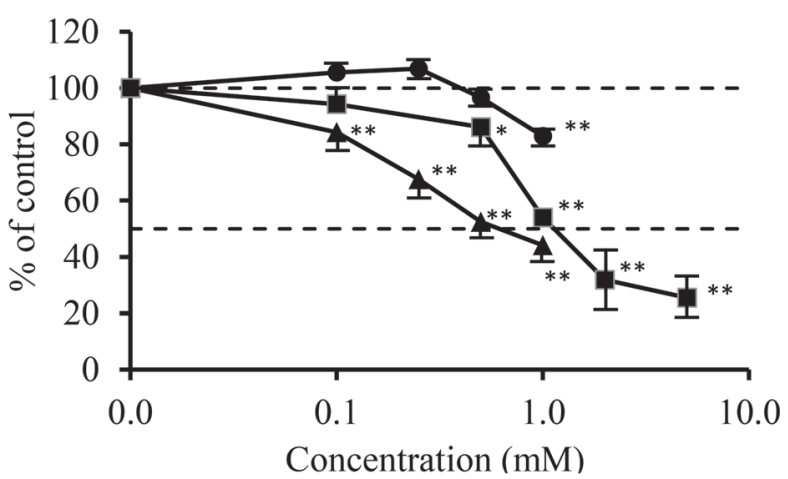

Fig. 1. Inhibitory effects of amoxicillin, ampicillin, and piperacillin against CYP2C8-mediated aminopyrine $\mathrm{N}$-demethylation activity. $\mathbf{\Lambda}$ : amoxicillin, $\bullet$ : ampicillin, $\mathbf{m}$ : piperacillin. Values are means \pm S.D. of triplicate experiments. $* p<0.05,{ }^{*} p<0.01$.

and piperacillin were 0.83 and $1.14 \mathrm{mM}$, respectively.

\section{DISCUSSION}

When the substrate concentration is lower than the $\mathrm{K}_{\mathrm{m}}$ value, the ratio of intrinsic clearance $\left(\mathrm{CL}_{\text {int }}\right)$ in the presence and absence of the inhibitor can be expressed by the following equation, independent of the inhibition type, except in the case of uncompetitive inhibition (Ito et al., 1998a, 1998b; Pharmaceuticals and Medical Devices Agency, 2001),

$$
\mathrm{CL}_{\text {int }}(+ \text { Inhibitor }) / \mathrm{CL}_{\text {int }}(- \text { Inhibitor })=1 /\left(1+\mathrm{I}_{\mathrm{u}} / \mathrm{K}_{\mathrm{i}}\right)
$$

where $I_{u}$ and $K_{i}$ are the unbound concentration of the inhibitor and the inhibition constant, respectively. $\mathrm{K}_{\mathrm{i}}$ values are expected to be equal to $\mathrm{IC}_{50} / 2$ or $\mathrm{IC}_{50}$ in competitive or noncompetitive inhibition, respectively, when the substrate concentrations are around the $\mathrm{K}_{\mathrm{m}}$. In addition, when the absorption rate is maximum, the maximum inflow concentration of the inhibitor into the liver $\left(\mathrm{I}_{\mathrm{in}, \max }\right)$ can be expressed as,

$$
\mathrm{I}_{\mathrm{in}, \max }=\mathrm{I}_{\text {max }} \cdot \mathrm{R}_{\mathrm{B}}+\left\{\left(\mathrm{k}_{\mathrm{a}} \cdot \mathrm{D} / \mathrm{Q}_{\mathrm{H}}\right) \cdot \mathrm{Fa}\right\}
$$

where $I_{\max }, R_{B}, k_{a}, D, Q_{H}$, and $F_{a}$ represent the maximum plasma concentration of the inhibitor in circulation, bloodplasma concentration ratio, absorption rate constant, dose, hepatic blood flow, and the fraction absorbed from the gastrointestinal tract into the portal vein, respectively. After an oral dosing of $250 \mathrm{mg}$ amoxicillin in healthy volunteers, the peak plasma concentration $\left(\mathrm{I}_{\max }\right)$ was
$3.68 \mu \mathrm{g} / \mathrm{mL}(8.8 \mu \mathrm{M})$ (Sawacillin Package Insert, 2015). Additionally, approximately $72.2 \%$ of dose was excreted in urine unchanged and no metabolites with antibacterial activity were detected in the serum or urine (Sawacillin Package Insert, 2015). $\mathrm{I}_{\mathrm{in} \text {,max }}$ for amoxicillin was calculated to be $46 \mu \mathrm{M}$ using $\mathrm{R}_{\mathrm{B}}=1, \mathrm{k}_{\mathrm{a}}=0.1 \mathrm{~min}^{-1}, \mathrm{Q}_{\mathrm{H}}=$ $1610 \mathrm{~mL} / \mathrm{min}, \mathrm{F}_{\mathrm{a}}=1$ to avoid false-negative predictions. Protein binding of amoxicillin in human serum was reported to be 16.6\%-25.0\%. (Sawacillin Package Insert, 2015). Therefore, the predicted free fractions of $I_{\text {in,max }}$ are much lower than the concentration investigated in this study (0.1-0.5 mM) and estimated $\mathrm{IC}_{50}$ for CYP2C8 $(0.83 \mathrm{mM})$, suggesting that amoxicillin might not affect drug pharmacokinetics and CYP2C8-, CYP2C9-, CYP2D6-, or CYP3A4/5-mediated drug metabolism. $I_{\max }$ of ampicillin after intravenous administration of $1 \mathrm{~g}$ ampicillin as sulbactam sodium-ampicillin sodium (Unasyn ${ }^{\circledR}$ ) and piperacillin after 30-min infusion of $4 \mathrm{~g}$ piperacillin as tazobactam sodium-piperacillin sodium for injection $\left(\right.$ Zosyn $\left.^{\circledR}\right)$ are $78.80 \mu \mathrm{g} / \mathrm{mL}(0.21 \mathrm{mM})$ and $286 \mu \mathrm{g} / \mathrm{mL}(0.53 \mathrm{mM}$ ), respectively (Zosyn Package Insert, 2015; Unasyn Package Insert, 2015). Serum/ plasma protein binding of ampicillin and piperacillin was low, $31.8 \%$ and $16 \%$, respectively (Zosyn Package Insert, 2015; Unasyn Package Insert, 2015). Thus, piperacillin as well as ampicillin might not affect drug pharmacokinetics and CYP2C8-, CYP2C9-, CYP2D6-, or CYP3A4/5-mediated drug metabolism, although piperacillin might inhibit CYP2C8 clinically. In this regard, however, drugs predominantly metabolized by CYP2C 8 alone are not known. Further clinical studies are required.

In conclusion, the present study suggests that piperacillin might inhibit CYP2C8 clinically although the interactions between the three penicillin-based antibiotics investigated and other drugs, which are metabolized by human hepatic drug-metabolizing P450s such as CYP2C8, CYP2C9, CYP2D6, and CYP3A4/5, are not clinically significant.

Conflict of interest---- The authors declare that there is no conflict of interest.

\section{REFERENCES}

Fuhr, U., Anders, E.M., Mahr, G., Sörgel, F. and Staib, A.H. (1992): Inhibitory potency of quinolone antibacterial agents against cytochrome P450IA2 activity in vivo and in vitro. Antimicrob. Agents Chemother., 36, 942-948.

Funae, Y., Kishimoto, W., Cho, T., Niwa, T. and Hiroi, T. (2003): CYP2D in the brain. Drug Metab. Pharmacokinet., 18, 337-349.

Imaoka, S., Yamada, T., Hiroi, T., Hayashi, K., Sakaki, T., 
Yabusaki, Y. and Funae, Y. (1996): Multiple forms of human P450 expressed in Saccharomyces cerevisiae. Systematic characterization and comparison with those of the rat. Biochem. Pharmacol., 51, 1041-1050.

Ito, K., Iwatsubo, T., Kanamitsu, S., Nakajima, Y. and Sugiyama, Y. (1998a): Quantitative prediction of in vivo drug clearance and drug interactions from in vitro data on metabolism, together with binding and transport. Annu. Rev. Pharmacol. Toxicol., 38, 461499.

Ito, K., Iwatsubo, T., Kanamitsu, S., Ueda, K., Suzuki, H. and Sugiyama, Y. (1998b): Prediction of pharmacokinetic alterations caused by drug-drug interactions: metabolic interaction in the liver. Pharmacol. Rev., 50, 387-412.

Niwa, T., Hiroi, T., Tsuzuki, D., Yamamoto, S., Narimatsu, S., Fukuda, T., Azuma, J. and Funae, Y. (2004): Effect of genetic polymorphism on the metabolism of endogenous neuroactive substances, progesterone and p-tyramine, catalyzed by CYP2D6. Mol. Brain Res., 129, 117-123.

Niwa, T., Inoue, S., Shiraga, T. and Takagi, A. (2005): No inhibition of cytochrome P450 activities in human liver microsomes by sulpiride, an antipsychotic drug. Biol. Pharm. Bull., 28, 188191.

Niwa, T., Murayama, N., Emoto, C. and Yamazaki, H. (2008): Comparison of kinetic parameters for drug oxidation rates and substrate inhibition potential mediated by cytochrome P450 3A4 and 3A5. Curr. Drug Metab., 9, 20-33.

Niwa, T., Murayama, N., Imagawa, Y. and Yamazaki, H. (2015): Regioselective hydroxylation of steroid hormones by human cytochromes P450. Drug Metab. Rev., 47, 89-110.

Niwa, T., Sato, R., Yabusaki, Y., Ishibashi, F. and Katagiri, M. (1999): Contribution of human hepatic cytochrome P450s and steroidogenic CYP17 to the N-demethylation of aminopyrine. Xenobiotica, 29, 187-193.

Niwa, T., Shiraga, T., Hashimoto, T. and Kagayama, A. (2004): Effect of cefixime and cefdinir, oral cephalosporins, on cyto- chrome P450 activities in human hepatic microsomes. Biol. Pharm. Bull., 27, 97-99.

Niwa, T., Yamamoto, S., Saito, M., Shiraga, T. and Takagi, A. (2007): Effect of cyclosporine and tacrolimus on cytochrome P450 activities in human liver microsomes. Yakugaku Zasshi, 127, 209-216.

Pharmaceuticals and Medical Devices Agency. Methods of drug interaction studies (PMSB/ELD Notification No. 813; June 4, 2001). http://www.nihs.go.jp/phar/pdf/DiGlEngFinal011209.pdf.

Rendic, S. (2002): Summary of information on human CYP enzymes: human P450 metabolism data. Drug Metab. Rev., 34, 83-448.

Sawacillin Package Insert, Astellas Pharma, January 2015, http:// www.info.pmda.go.jp/go/pack/6131001C1210_1_12/

Shimada, T., Yamazaki, H., Mimura, M., Inui, Y. and Guengerich, F.P. (1994): Interindividual variations in human liver cytochrome P-450 enzymes involved in the oxidation of drugs, carcinogens and toxic chemicals: studies with liver microsomes of 30 Japanese and 30 Caucasians. J. Pharmacol. Exp. Ther., 270, 414-423.

Unasyn Package Insert, Pfizer Japan Inc., June 2015, http://www.info. pmda.go.jp/downfiles/ph/PDF/671450_6139504G1028_2_05. pdf

von Rosensteil, N.A. and Adam, D. (1995): Macrolide antibacterials. Drug interactions of clinical significance. Drug Saf., 13, 105-122.

Williams, J.A., Hyland, R., Jones, B.C., Smith, D.A., Hurst, S., Goosen, T.C., Peterkin, V., Koup, J.R. and Ball, S.E. (2004): Drug-drug interactions for UDP-glucuronosyltransferase substrates: a pharmacokinetic explanation for typically observed low exposure (AUCi/AUC) ratios. Drug Metab. Dispos., 32, 1201-1208.

Wright, A.J. (1999): The penicillins. Mayo Clin. Proc., 74, 290-307.

Zosyn Package Insert, Taisho Toyama Pharmaceutical Co., Ltd., June 2015, http://www.info.pmda.go.jp/go/pack/6139505F3020_1_07/ 